\title{
Nanomold Fabrication by Scratching and Its Application to Nanoimprint Lithography
}

\author{
Jun SHIMIZU ${ }^{1}$, Wataru OHSONE ${ }^{2}$, Hirotaka OJIMA ${ }^{3}$, Teppei ONUKI ${ }^{4}$, Libo ZHOU ${ }^{5}$ and Takeyuki YAMAMOTO \\ ${ }^{1}$ Department of Intelligent Systems Engineering, Ibaraki University, Japan, jshimizu@mx.ibaraki.ac.jp \\ ${ }^{2}$ Graduate School of Science and Engineering, Ibaraki University, Japan, jshimizu@mx.ibaraki.ac.jp \\ ${ }^{3}$ Department of Intelligent Systems Engineering, Ibaraki University, Japan, ojima@mx.ibaraki.ac.jp \\ ${ }^{4}$ Department of Intelligent Systems Engineering, Ibaraki University, Japan, onuki-t@mx.ibaraki.ac.jp \\ ${ }^{5}$ Department of Intelligent Systems Engineering, Ibaraki University, Japan, lbzhou@mx.ibaraki.ac.jp \\ ${ }^{6}$ Department of Intelligent Systems Engineering, Ibaraki University, Japan, ytake@mx.ibaraki.ac.jp
}

\begin{abstract}
:
This study aimed to fabricate nanoscale structures on monocrystalline silicon substrates using nanoscratching. In this paper, nano/micro-scale line-and-space patterns were generated on a silicon substrate using an atomic force microscope equipped with a sharp probe made of monocrystalline diamond. Subsequent chemical etching was also conducted on the fabricated line-and-space patterns. As a result, it was confirmed that the groove was deepened several times but little increased in its width. A nanoimprint experiment was also performed to duplicate the line-and-space patterns by use of the fabricated line-and-space mold and polycarbonate resin film.
\end{abstract}

Keywords: Nanoscratching, Mold, Silicon wafer, Diamond probe, Chemical etching, Nanoimprint

\section{Introduction}

With the advances in the miniaturizations of electron, optical and magnetic devices, their manufacturing methods have become more precise and complicated. The current processes are mainly dependent on the MEMS technologies. These processes require complicated and multistage facilities, thus are extremely costly. Therefore, alternative patterning methods, which are more concise, economic and precise, need to be developed to meet the increasingly high demand from manufacturing industries. Nanoimprint lithography (NIL) [1] is one of the potential solutions. NIL is a novel and simple method capable of producing nano-scale patterns on resin or glass using nano/micro molds similar to usual injection moldings. This technology has been used to fabricate nanostructures for antireflection on LCD (liquid crystal display) panel, optical waveguide, and so on. To date, the nano/micro molds are mainly fabricated using photolithography or focused ion beams. However, the beam methods normally require special instruments and take a long time for drawing precise patterns.

Under such circumstances, our research aimed to fabricate various nano-patterns using nanoscratching [2-4], which is considered as one of the simplest methods for nano/micro molds fabrication. Although there were some attempts to examine the chip removal and material deformation mechanisms reported for monocrystalline silicon [5-6], few researches have been focused on the combination of mechanical and chemical methods [3,7]. In this paper, several nanoscratching experiments were performed to generate nano/micro-scale line-and-space patterns on a monocrystalline silicon substrate using an atomic force microscope (AFM) equipped with a sharp diamond probe. Subsequent chemical etching was also conducted on the fabricated groove patterns to enlarge aspect ratio. Nanoimprinting test was also performed using fabricated mold for line-and-space patterns duplication.

\section{Nanoscratching Methods and Conditions}

Nanoscratching experiments were conducted with a scanning probe microscope (SPM), which has the function of environmental control (SPA-300HV, Seico Instruments Inc.). In the experiments, the AFM mode (or contact mode) was utilized for both the scratching and surface measurement with a constant normal load. The probe was made of monocrystalline diamond and in three sided pyramid, as shown in Fig. 1. The scratching tests were performed by either front face or back face (edge) of the diamond probe.

Table 1 shows the experimental conditions for the present nanoscratching tests. The same diamond probe was also used to measure profiles of the scratch grooves to ease finding the scratched grooves.

A Si(100) bare wafer finished by the commercial CMP (chemical mechanical polishing) technique was used as the workpiece. The wafer was washed in acetone and ethanol in an ultrasonic cleaner, and then rinsed in pure water prior to nanoscratching.

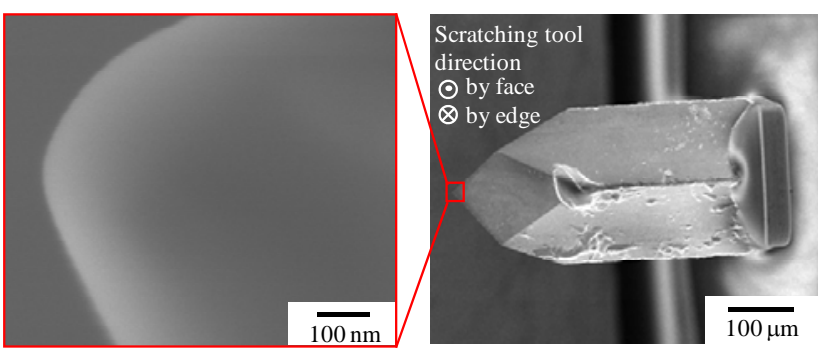

Figure 1: SEM photos of diamond probe 
Table 1: Experimental conditions in nanoscratching

\begin{tabular}{|l|l|}
\hline Experimental apparatus & SPA-300HV (SII) \\
\hline Workpiece & $\mathrm{Si}(100)$ wafer \\
\hline Scratching/observing tool & Monocrystalline diamond \\
\hline Cantilever stiffness & $200 \mathrm{~N} / \mathrm{m}$ \\
\hline Diamond tip radius & $100 \mathrm{~nm}$ (initial value) \\
\hline Three edge angle & $80 \mathrm{deg}$. \\
\hline Environment & $\mathrm{RT}(297 \mathrm{~K})$ in air \\
\hline Scratching speed & $1 \mu \mathrm{m} / \mathrm{s}$ \\
\hline Scratching direction & $<110>$ \\
\hline Measurement load & $20 \mathrm{nN}$ \\
\hline
\end{tabular}

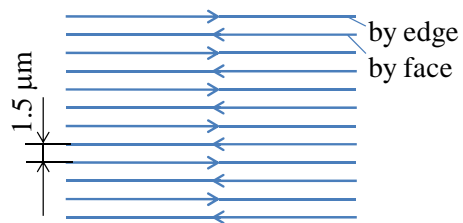

(a) Illustration of scratching patterns arrangement

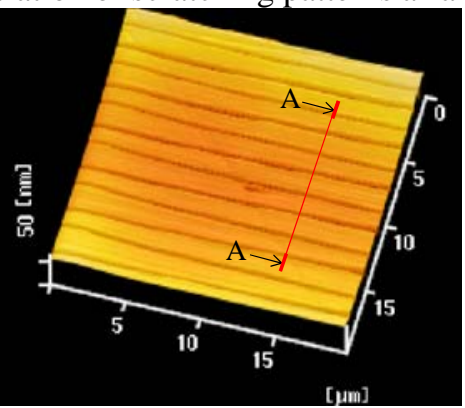

(b) Bird-eye view

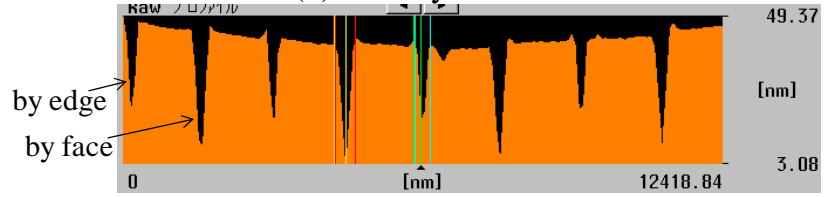

(c) Cross-sectional view

Figure 2: (a) Illustration of scratch arrangement. AFM images of the scratched grooves: (b) Bird-eye view and (c) cross-sectional view. A face and edge of diamond tool was used and the normal load for scratching was $30 \mu \mathrm{N}$.

\section{Nanoscratching Methods and Conditions}

\subsection{Influence of Scratching Tool Direction and Normal Load on Groove Geometry}

In order to understand the influence of scratching tool direction (face or edge) and normal load on scratching groove geometry, a series of scratching tests were alternatively conducted by tool face and edge by varying normal loads. Figure 2(a) shows the details of scratching procedures. The example shown in Figs. 2(b) and (c) are the bird-eye and cross-sectional views of the grooves scratched at the normal load of $30 \mu \mathrm{N}$.

From Fig. 2, it can be seen that all the grooves generated have sharp tips, which suggests successful transcription of the tool tip shape. Small burrs of several nanometers in height are observed on the groove shoulders. The grooves fabricated by the face scratching are deeper than those made by the edge scratching. The grooves under the same scratching condition have almost equal depths. This trend shows the stability of the present nanoscratching tests.

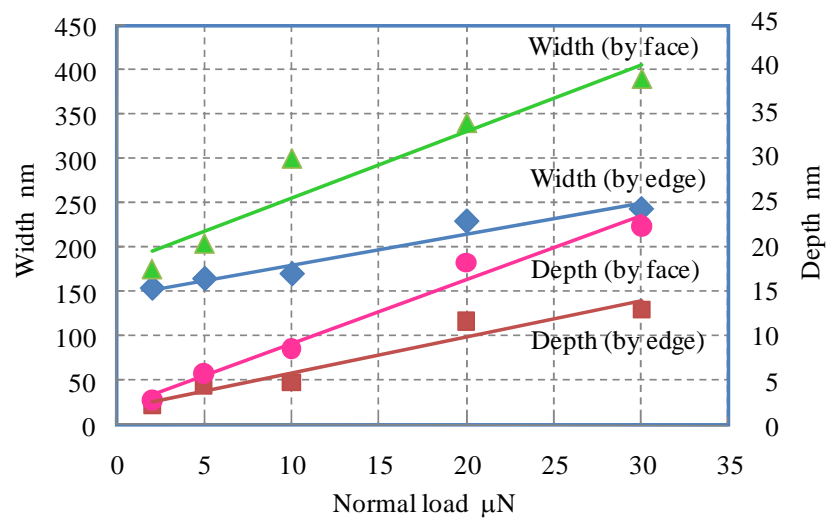

Figure 3: Effect of scratching tool direction and normal load on groove width and depth

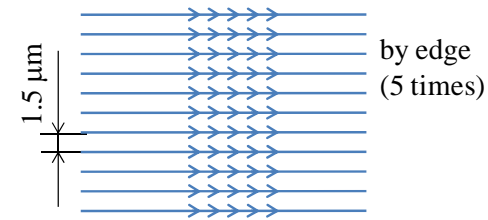

(a) Illustration of scratching patterns arrangement

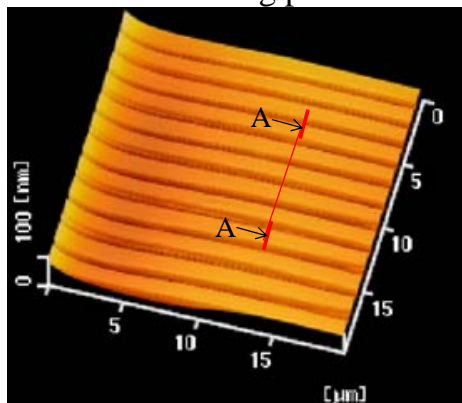

(b) Bird-eye view

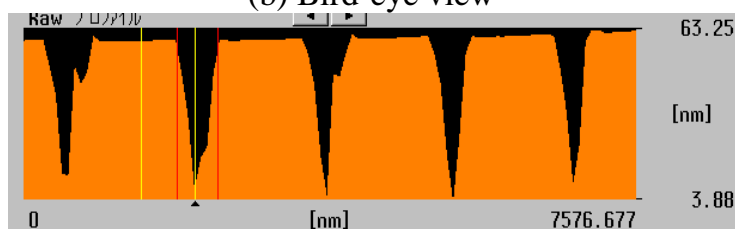

(c) Cross-sectional view

Figure 4: (a) Illustration of scratch arrangement. AFM images of line-and-space patterns: (b) Bird-eye view and (c) cross-sectional view. An edge of diamond tool was used and each single groove was generated by quintuple scratches with $10 \mu \mathrm{N}$ in normal load.

The width and depth of the scratch grooves are plotted as a function of the normal load in Fig. 3. It is clear that both width and depth of the grooves made by face scratching are almost twice as those by edge scratching. The width and depth of the grooves are increasing linearly with the normal scratching load. These results suggested that the present nanoscratching tests were consistent.

\subsection{Fabrication of Line-and-space Patterns}

Figure 4 shows an example of fabricated line-and-space patterns by edge scratching. As shown in Fig. 4(a), each single groove is generated by quintuple scratches with 10 $\mu \mathrm{N}$ of normal load. From Fig. 4(b), each line and space (groove and unscratched area) can be clearly recognized. From Fig. 4(c), it can be observed that the width of each line and space are about $500 \mathrm{~nm}$ and $1000 \mathrm{~nm}$, and the 
Table 2: Etching conditions after nanoscratching

\begin{tabular}{|l|l|}
\hline Workpiece & Line-and-space patterns \\
\hline Etchant & KOH solution (5 and 30 \%) \\
\hline Additive in etchant & $\begin{array}{l}\text { Water-soluble non-ionic } \\
\text { surfactant (0.1 \%) }\end{array}$ \\
\hline Etching time & 50 minutes \\
\hline Environment & Room temperature (297 K) \\
\hline
\end{tabular}

average groove depth is about $50 \mathrm{~nm}$, respectively. The width and depth become about 3 and 10 times large compared that of the single scratch at the same normal load of $10 \mu \mathrm{N}$ shown in Fig. 2. From this result, it is grasped that the groove width and depth can be enlarged by multiple scratching.

\subsection{Improvement by Subsequent Chemical Etching}

In the case of the alkaline etching of monocrystalline silicon by $\mathrm{KOH}$ solution, the chemical reaction equations are expressed as follows [8]:

$$
\begin{aligned}
& \mathrm{Si}+4 \mathrm{OH}^{-} \rightarrow \mathrm{Si}(\mathrm{OH})_{4}+4 \mathrm{e}^{-} \\
& 4 \mathrm{H}_{2} \mathrm{O}+4 \mathrm{e}^{-} \rightarrow 4 \mathrm{OH}^{-}+2 \mathrm{H}_{2}
\end{aligned}
$$

These equations indicate that the silicon is directly etched by alkaline solutions. On the other hand, the amorphous silicon layer generated by the nanoscratching without cutting chip formation under relatively low normal loads showed the masking effect for alkaline solutions, while such a phenomenon was hardly recognized under higher normal loads [3,7].

From these facts, it can be expected that the grooves depths after relatively high-load scratching with cutting chip formation, are increased by the alkaline etching, because the defects such as dislocations generated beneath the groove due to relatively large deformation, behave as the pathways of hydroxyl ions and locally enhance the etching rate for monocrystalline silicon.

In order to obtain deeper or higher aspect ratio groove patterns, the chemical etching was conducted on the fabricated line-and-space molds by alkaline solutions. Table 2 shows the etching conditions. Two line-and-space molds fabricated under the same conditions shown in Fig. 5, were etched by 5 and $30 \%$ solutions of $\mathrm{KOH}$ for 50 minutes.

Figures 5(a),(b) and 6 show the laser micrographs of the etched groove patterns and the comparison result of groove geometries before and after etching, respectively. In the measurements, a laser microscope (OLS4000-SMT made by Olympus Corporation) was used.

From these results, it is understood that both the largest depth and aspect ratio are achieved by using $30 \%$ solutions of $\mathrm{KOH}$, while the increase in the width is insignificant. Particularly, the aspect ratio is increased from 0.1 to 0.4 , and this is quadruple. This result also means that the further deeper grooves can be obtained without remarkable increase in the groove width by introducing the proper chemical etching after scratching.

\section{Nanoimprint Lithography Experiment}

\subsection{Experimental Setup and Conditions}

Nanoimprint lithography experiments were conducted using a simple self-developed apparatus under the
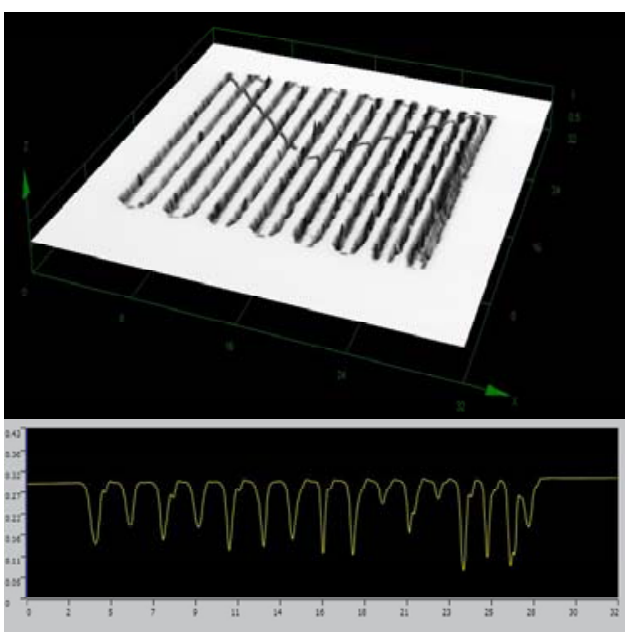

(a) $\mathrm{KOH} 5 \%$

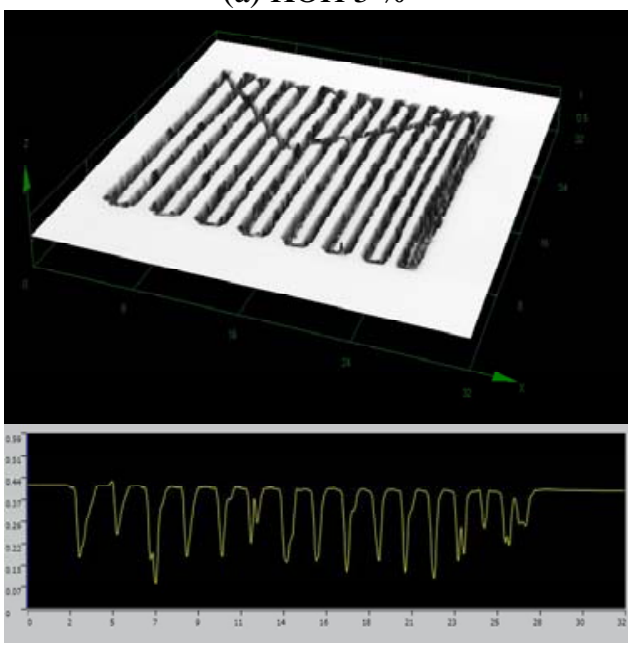

(b) $\mathrm{KOH} 30 \%$

Figure 5: Laser micrographs of etched line-and-space patterns shown in Fig. 4

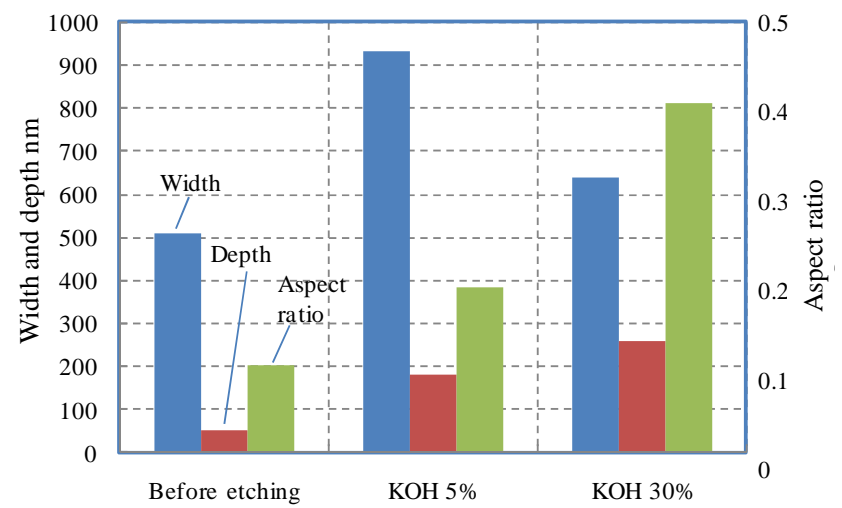

Figure 6: Comparison result of groove geometries before and after etching

conditions shown in Table 3. A heated mold at $300^{\circ} \mathrm{C}$ was pressed against a polycarbonate resin film at the pressure of $16 \mathrm{MPa}$ for 10 minutes. A mold with line-and-space patterns, which were created by face scratching and followed by chemical etching, was used.

\subsection{Experimental Setup and Conditions}

Figure 7(a) shows the laser micrographs of fabricated mold and (b) imprinted polycarbonate resin workpiece, respectively. Also, Fig. 8 shows the comparison result of average pattern geometries between mold and imprinted polycarbonate resin. 
Table 3: Conditions for nanoimprinting test

\begin{tabular}{|l|l|}
\hline Mold & $\begin{array}{l}\text { Line-and-space patterns } \\
\text { etched by } 30 \% \mathrm{KOH} \\
\text { solution for } 10 \text { minutes }\end{array}$ \\
\hline Workpiece & Polycarbonate resin film \\
\hline Heat temperature of mold & $573 \mathrm{~K}$ \\
\hline Mold release agent & $\begin{array}{l}\text { SAM (Fluorinated silane } \\
\text { coupling agent) }\end{array}$ \\
\hline Applied pressure & $16 \mathrm{MPa}$ \\
\hline Holding time & 10 minutes \\
\hline $\begin{array}{l}\text { Coating material for } \\
\text { measurement }\end{array}$ & Pt sputter coating \\
\hline
\end{tabular}

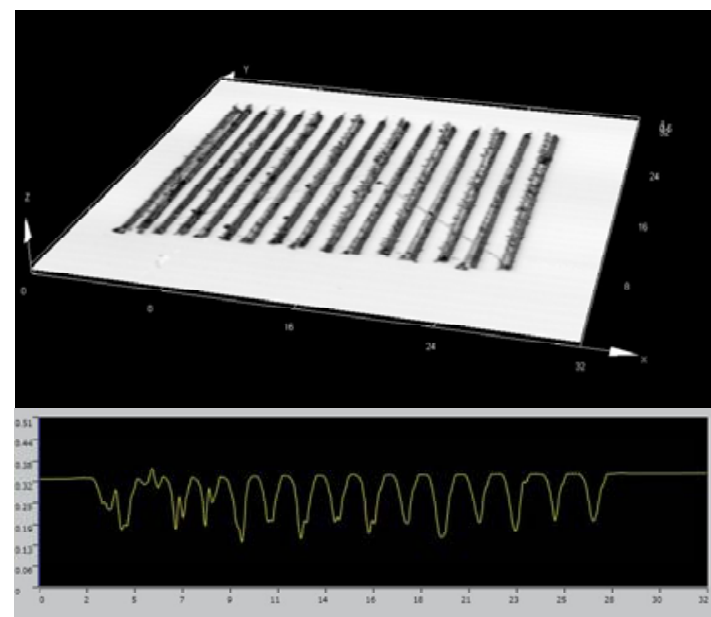

(a) Mold patterns
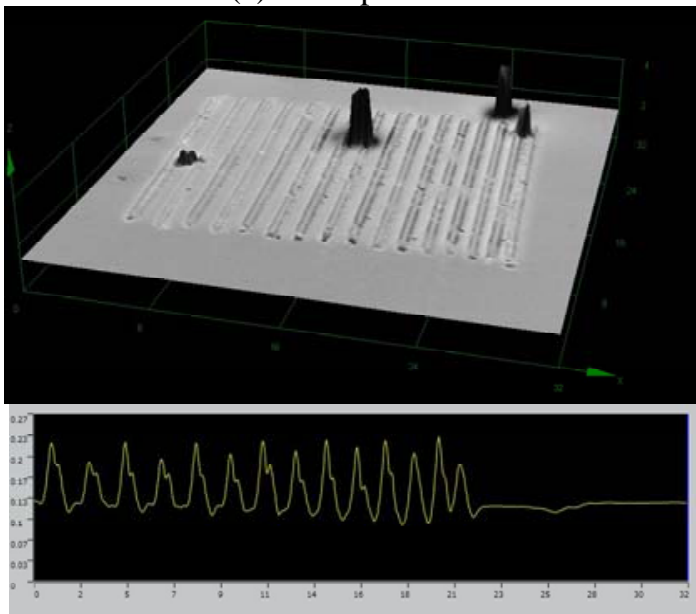

(b) Replicated patterns

Figure 7: Laser micrographs of (a) mold and (b) replicated patterns on polycarbonate resin

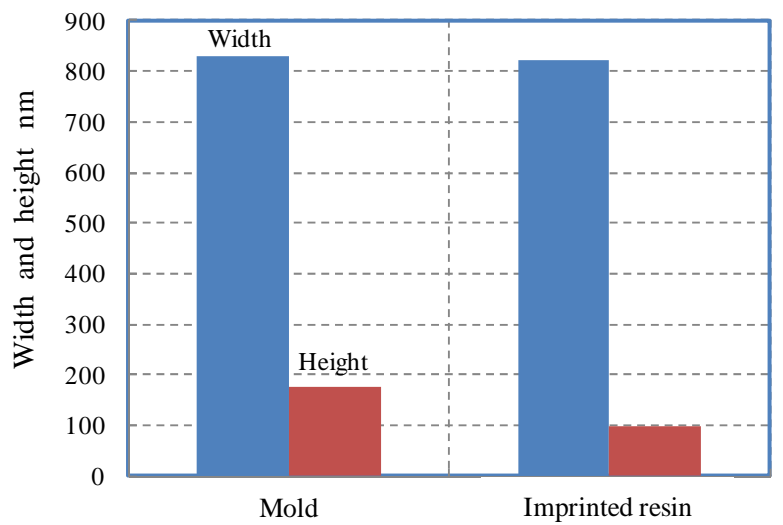

Figure 8: Comparison result of average pattern geometries between mold and duplicated patterns
From these results, it can be seen that the average height of the protrusion patterns is around $98 \mathrm{~nm}$, which is $55 \%$ of the average groove depth of the mold patterns $(178 \mathrm{~nm})$, while the average width is $824 \mathrm{~nm}$, which is almost the same as that of the mold patterns $(829 \mathrm{~nm})$. Such a shortage in imprinted protrusion height could be dissolved by increasing the normal load and/or introducing a vacuum environment.

\section{Conclusion}

Nano-scale structures for nanoimpriting were fabricated by the nanoscratching on monocrystalline silicon substrates using an atomic force microscope equipped with a sharply pointed monocrystalline diamond probe. As a result, line-and-space patterns were generated almost successfully. It was also clarified that higher groove aspect ratio can be achieved by combining the nanoscratching and alkaline etching. The line-and-space patterns were also imprinted almost successfully on the polycarbonate resin film by using the fabricated mold.

\section{Acknowledgement}

This work was partially supported by Grant-in-Aid for Fundamental Research (C) (No. 22560102 and No. 22560134) from Japan Society for the Promotion of Science.

\section{References}

[1] Chou, S.Y., Krauss, P. R. and Renstrom, P. J., 1995, Imprint of Sub-25 nm Vias and Trenches in Polymers, Phy. Rev. A, Vol. 67, pp. 3114-3116.

[2] Ashida, K., Morita, N. and Yoshida, Y., 2001, Study on nano-machining process using mechanism of a friction force microscope, JSME Int. J. (Series C), Vol. 44, pp. 244-253.

[3] Park, J. W., Kawasegi, N., Morita, N. and Lee, D. W., 2004, Tribo-nanolithography of silicon in aqueous solution based on atomic force microscope, Appl. Phys. Lett., Vol. 85, pp. 1766-1768.

[4] Youn, S. W. and Kang, C. G., 2006, Effect of nanoscratch conditions on both deformation behavior and wet-etching characteristics of silicon (100) surface, Wear, Vol. 261, pp. 328-337.

[5] Yoshino, M., Aoki, T., Shirakashi T. and Komanduri, R., 2001, Some experiments on the scratching of silicon: In situ scratching inside an SEM and scratching under high external hydrostatic pressures, Int. J. Mech. Sci., Vol. 43, pp. 335-347.

[6] Wu, Y. Q., Huang, H., Zou, J. and Dell, J. M., 2009, Nanoscratch-induced deformation of single crystal silicon, J. Vac. Sci. \& Technol. B, Vol. 27, pp. 1374-1377.

[7] Kawasegi, N. and Morita, N., 2010, High-AspectRatio Structure Fabrication on (110)-Oriented Silicon Surfaces Using Tribo-Nanolithography, J. Nanosci. and Nanothechnol., Vol.10, pp. 2394-2400.

[8] Seidel, H., Csepregi, L., Heuberger, A., Baumgartel, H. and Electrochem, J., 1990, Anisotropic Etching of Crystalline Silicon in Alkaline Solutions, J. Electrochemical Soc., Vol.137, pp. 3612-3626. 